\title{
Keck Interferometer Observations of FU Orionis Objects
}

\author{
R. Millan-Gabet ${ }^{1}$, J. D. Monnier ${ }^{2}$, R. L. Akeson ${ }^{1}$, L. Hartmann³ , J.-P. Berger ${ }^{4}$, \\ A. Tannirkulam ${ }^{2}$, S. Melnikov ${ }^{5}$, R. Billmeier ${ }^{2}$, N. Calvet ${ }^{3}$, P. D'Alessio $^{6}$, L. A. Hillenbrand ${ }^{7}$, \\ M. Kuchner ${ }^{8}$, W. A. Traub ${ }^{3}$, P. G. Tuthill ${ }^{9}$, C. Beichman ${ }^{1}$, A. Boden ${ }^{1}$, A. Booth ${ }^{10}$, \\ M. Colavita ${ }^{10}$, M. Creech-Eakman ${ }^{10}$, J. Gathright ${ }^{11}$, M. Hrynevych ${ }^{11}$, C. Koresko ${ }^{1}$, \\ D. Le Mignant ${ }^{11}$, R. Ligon ${ }^{10}$, B. Mennesson ${ }^{10}$, C. Neyman ${ }^{11}$, A. Sargent ${ }^{7}$, M. Shao ${ }^{10}$, \\ M. Swain ${ }^{4}$, R. Thompson ${ }^{1}$, S. Unwin ${ }^{10}$, G. van Belle ${ }^{1}$ G. Vasisht ${ }^{10}$, \& P. Wizinowich ${ }^{11}$ \\ rafael@ipac.caltech.edu
}

\begin{abstract}
We present new K-band long baseline interferometer observations of three young stellar objects of the FU Orionis class, V1057 Cyg, V1515 Cyg and Z CMa-SE, obtained at the Keck Interferometer during its commissioning science period. The interferometer clearly resolves the source of near-infrared emission in all three objects. Using simple geometrical models we derive size scales $(0.5-4.5 \mathrm{AU})$ for this emission. All three objects appear significantly more resolved than expected from simple models of accretion disks tuned to fit the broadband optical and infrared spectro-photometry. We explore variations in the key parameters that are able to lower the predicted visibility amplitudes to the measured levels, and conclude that accretion disks alone do not reproduce the spectral energy distributions and K-band

\footnotetext{
${ }^{1}$ Michelson Science Center, California Institute of Technology, MS 100-22, Pasadena, CA 91125

${ }^{2}$ University of Michigan Astronomy Department, Ann Arbor, MI 48109-1090, USA.

${ }^{3}$ Harvard-Smithsonian Center for Astrophysics, Cambridge, MA 02138, USA

${ }^{4}$ Laboratoire d'Astrophysique de Grenoble, 414 Rue de la Piscine 38400 Saint Martin d'Heres, France

${ }^{5}$ Ulugh Beg Astronomical Institute, Tashkent 700052, Uzbekistan

${ }^{6}$ Universidad Nacional Autónoma de México

${ }^{7}$ Astronomy Department, California Institute of Technology, Pasadena, CA

${ }^{8}$ Princeton University, Princeton, NJ

${ }^{9}$ University of Sydney, Physics Department

${ }^{10}$ Jet Propulsion Laboratory, California Institute of Technology, Pasadena, CA 91109

${ }^{11}$ W. M. Keck Observatory, California Association for Research in Astronomy, Kamuela, HI 96743
} 
visibilities simultaneously. We conclude that either disk models are inadequate to describe the near-infrared emission, or additional source components are needed. We hypothesize that large scale emission (10s of AU) in the interferometer field of view is responsible for the surprisingly low visibilities. This emission may arise in scattering by large envelopes believed to surround these objects.

\section{Introduction}

FU Orionis objects are a small but remarkable class of stars that display severalmagnitude outbursts in visible light, followed by decade-long fading phases. They also exhibit fluxes in excess of photospheric levels at infrared wavelengths, broad and doubled spectral absorption lines, and wavelenght-dependent rotational velocities. Two main theories compete to explain their nature. Herbig, Petrov \& Duemmler (2003) (and references therein) favor a model in which the flare occurs in an unstable star rotating near breakup, and where the spectral properties are explained by a rapidly rotating $\mathrm{G}$-supergiant photosphere overlaid with a rising cooler shell. In contrast, a more widely accepted view first proposed by Hartmann \& Kenyon (1985), is that FU Orionis stars are pre-main sequence objects and that all the observables have their origin in Keplerian circumstellar disks and pre-natal infalling envelopes that surround them (see also Hartmann, Hinkle \& Calvet 2004, for recent arguments in relation to the disk vs. rapidly rotating star debate). In this picture, the outbursts have their origin in sudden increases in the accretion rate through the disk, and all young stars may experience (probably recurrent) FU Orionis phases during their evolution (see also the review by Hartmann \& Kenyon 1996).

Although the existence of circumstellar disks around young stars is well established observationally, very little is directly known about the structure and properties of the innermost disk regions $(\lesssim 1 \mathrm{AU})$. For solar-mass and intermediate mass young stars, disk models have been reasonably successful at reproducing the infrared flux excesses characteristic of these systems, as well as the spectroscopic signatures of the accretion process itself. However, these efforts are fundamentally limited by the fact that the observations are spatially unresolved, which introduces important degeneracies with respect to the spatial distribution of the emitting material.

The degeneracies with respect to the spatial distribution of material inherent in spatially unresolved observations can be lifted by observations using a long baseline interferometer, and important progress has already been made using this technique. For Herbig Ae/Be and $\mathrm{T}$ Tauri objects (young stars of intermediate and solar-type mass respectively), all observations to date have revealed surprisingly large spatial scales for the near-infrared (NIR) 
emission (Millan-Gabet et al. 1999; Tuthill, Danchi \& Monnier 1999; Akeson et al. 2000; Millan-Gabet, Schloerb, and Traub 2001; Akeson et al. 2002; Eisner et al. 2004; Akeson et al. 2005; Eisner et al. 2005; Akeson et al. 2005b; Monnier et al. 2005); prompting in part a significant revision of disk models, whereby previously ignored detailed physics of the disk inner edge are now incorporated with some success (Natta et al. 2001; Dullemond, Dominik \& Natta 2001; Monnier \& Millan-Gabet 2002; Muzerolle et al. 2003; Vinković et al. 2003). For a review of observational breakthroughs in this area recently made possible by interferometric techniques at infrared wavelengths, the reader is also referred to Millan-Gabet et al. (2006).

For FU Orionis objects, far fewer direct observational constraints exist at those scales. On the other hand, following the initial outburst the disk emission in FU Orionis objects outshines that of their central stars by many orders of magnitude, making them in principle ideal laboratories for testing theories describing the structure and physical properties of accretion disks. Indeed, the interpretation is freed from several complicating factors that affect the more evolved Herbig Ae/Be and T Tauri systems: instead of having multiple source components (star plus disk) and disk heating mechanisms (stellar irradiation plus active accretion), only the disk component and active accretion matters for interpreting observations of FU Orionis objects. Given that these same theories are being applied to the disks inferred to exist around all young stars, and that the physical conditions in the inner parts of those disks set the initial conditions for planet formation, it is important to validate the disk paradigm using FU Orionis objects as ground truth tests.

The very first young stellar object to be resolved by long baseline optical interferometry was in fact FU Orionis itself, and in their original analysis and follow up work Malbet et al. $(1998,2005)$ concluded that the visibility amplitudes measured agreed well with those predicted by thermal emission in an accretion disk with the canonical temperature profile $T \sim r^{-3 / 4}$ with parameters tuned to fit the spectral energy distribution (SED).

In this paper we present new interferometer data on three other well known FU Orionis objects, and find instead that the NIR emission is over-resolved relative to predictions of models that consist only of a standard disk emitting thermally. In $\S 2$ we present the observations, instrumental arrangement and data analysis; in $\S 3.1$ we derive spatial scales for the NIR emission using simple geometrical models of general applicability; in $\S 3.3$ we construct accretion disk models with which we attempt to simultaneously fit the broadband SEDs and visibility amplitude data; in $\S 3.4$ we consider additional source components that may be required in order to explain the measured visibilities; and in $\S 4$ we discuss our results in the broader context and suggest future lines of investigation. 


\section{Observations and Data Analysis}

We have observed three well studied FU Orionis objects: V1057 Cyg, V1515 Cyg and the South-East component of the binary system Z CMa. Basic properties of these objects are given in Table 1. We note that the photometry data given in this table is meant to inform about the instrumental conditions only; photometry used for modelling purposes was constructed as described in $\S 3.2$.

These observations were made at the Keck Interferometer (KI) during its visibility science commissioning period (2002-2004). The KI (Colavita, Wizinowich \& Akeson 2004) is formed by the two 10-m aperture Keck telescopes separated by $85 \mathrm{~m}$ along a direction $\sim 38$ degrees East of North, corresponding to a minimum fringe spacing of 5.3 milli-arcseconds (mas) at $2.2 \mu \mathrm{m}$. In order to coherently combine the NIR light from such large apertures, each telescope utilizes a natural guide star adaptive optics system (Wizinowich et al. 2003). Optical delay lines correct for sidereal motion and the telescope beams are combined at a beamsplitter before the light is focused onto single-mode (fluoride) fibers which impose a $~ 50$ mas (FWHM) field-of-view (FOV) for all data reported herein. While both $\mathrm{H}$ and K-band observations are now possible, only broad K-band $(2.18 \mu \mathrm{m}, \Delta \lambda=0.3 \mu \mathrm{m})$ data are reported here. For the four nights over which the data presented here were taken, observing conditions could be characterized as average, with fringe phase noise ("jitter", a measure of atmospheric seeing + instrument noise) in the range $0.5-1.25 \mathrm{rad}$, and constant for calibrators and targets. The system visibility, observed on calibrator point sources, was always normal $\left(V_{\text {system }}^{2}=0.6-0.7\right)$.

We note that, although the angular separation of the components in the Z CMa system is only $\sim 0.1^{\prime \prime}$ (Koresko et al. 1991), the adaptive optics sub-systems at KI clearly resolve the system, such that either binary component can be selected for interferometer measurements. Therefore, for the data presented here, the instrument has isolated the contribution from the South-East component, believed to be a FU Orionis object (Hartmann et al. 1989; Koresko et al. 1991). The North-West component is believed to be a high-mass young star surrounded by a dusty envelope (see e.g. Garcia, Thiebaut \& Bacon 1999) and was also resolved by

$\mathrm{KI}$; these results are discussed in Monnier et al. (2005). With this method, and given the relatively small angular distance between the two components, it is important to be sure that (1) the fringe detector was pointed at the intended component, and (2) received no extra flux from the other one. We have performed several checks to verify this. First, the mean K-band fluxes measured from each component are in a ratio (the Herbig component being $\sim \times 3$ brighter than the FU Ori component) that approximately agrees with (variable) values found in the literature (see references above). Second, we have verified that the fringes for each component were found at different optical delays, which differ by amounts 
consistent with the $\sim 0.1^{\prime \prime}$ astrometric offset. Finally, the single-mode fibers which feed the fringe camera impose a FOV ( $\sim 50$ mas as mentioned above) with an approximately Gaussian tapering. Therefore, even if assuming a $\times 3$ higher flux on the (off-axis) Herbig component, its flux contribution to the on-axis beam is less than $1 \%$, contributing negligibly to the measured visibilities. Moreover, the single-beam fluxes fluctuate by $30 \%$ or less, very consistently for all objects (the ZCMa components and the three calibrators used), indicating that uncompensated tip-tilt angle fluctuations are much less than one FWHM, and again implying a small fraction of flux contamination by ZCMa-NW to the ZCMa-SE measurement.

The square of the fringe visibility $\left(\mathrm{V}^{2}\right)$ was measured using the ABCD-method (using a dither mirror; see also Shao \& Staelin 1977) and we followed well-tested strategies described for the Palomar Testbed Interferometer (Colavita 1999), except that corrections for unbalanced telescope fluxes were improved, and jitter corrections were not applied. These forms of jitter corrections are relevant when optical path fluctuations are dominated by atmospheric residuals outside the fringe tracker bandwidth. At KI, these fluctuations are dominated by air motions in the Coude path and do not obey the same statistics, and detailed tests have shown that standard jitter corrections do not improve the data calibration. Calibration of fringe data was performed by interspersing target observations with those of unresolved calibrators; basic information on the calibrator stars used is given in Table 2. We note that although the calibrator stars are significantly brighter than the target FU Ori objects at visual wavelengths where the AO correction is performed, calibration tests (described in Akeson et al. 2005b) have shown that no systematic effects are introduced. Further details on the data reduction and calibration procedures may be found in Colavita et al. (2003) and Swain et al. $(2003)^{1}$.

The calibrated $\mathrm{V}^{2}$ results appear in Table 3 along with the projected baseline $(u, v)$, date of each observation and calibrator information. The $\mathrm{V}^{2}$ errors reported in Table 3 only include statistical errors. Internal data quality checks have established a conservative upper limit to the systematic error $\Delta V^{2}=0.05$, which we add in quadrature and include in all model fitting (and figures) presented in this paper.

\footnotetext{
${ }^{1}$ As well as in a series of technical memos at the Michelson Science Center (http://msc.caltech.edu/KISupport/)
} 


\section{Results}

\subsection{Characteristic Sizes of the Near-Infrared Emission}

As can been seen in Table 3, all three sources are clearly resolved by the KI (calibrated $\left.V^{2}<1.0\right)$. Since the complex visibility is related to the source brightness by a Fourier transform relation (see e.g. Goodman 1985), we can use a simple a priori representation of the object morphology and fit to the measured visibility amplitudes in order to extract source parameters of astrophysical value. This approach has the merit of providing fundamental

new information about the NIR emission (size scale) that is independent of (often poorly constrained) details of specific physical models.

We use two plausible geometric representations for the NIR brightness: a Gaussian and a uniform ring. The parameters to be fit are the Gaussian full width at half-maximum (FWHM) and the ring diameter respectively (the ring thickness is poorly constrained by measurements in the first lobe of the visibility function, and following previous work - e.g. Monnier et al. (2005) - we adopt a $20 \%$ fractional ring width). These results are given in Table 4. We note that in our sample, the only object previously resolved at these scales is V1057 Cyg, for which Wilkin \& Akeson (2003) similarly derive a Gaussian diameter that is larger than ours but within errors.

In the next sections we test the detailed predictions of accretion disk models, against both our new visibility measurements and reconstructed broadband SEDs.

\subsection{Constructing the SEDs}

It is not the purpose of this paper to perform detailed SED modelling, nor do our main conclusions depend on it. However, since FU Orionis objects are by definition photometrically variable, we have taken some care to construct meaningful (i.e. as nearcontemporaneous as possible) SEDs from existing data, as follows:

V1057 Cyg: We use near-contemporaneous UBVR data provided by one of us (Melnikov) and obtained in July and August 2002 at the Maidanak High Altitude Observatory as part of long term monitoring campaign of young stars (the ROTOR project, Herbst \& Shevchenko (1999); Clarke et al. (2005)). I-band data is interpolated between Kopatskaya et al. (2002) and photometry obtained by one of us (Tannirkulam) at the MDM observatory in November-December 2004. The NIR JHK photometry is from 2MASS. Abraham et al. (2004) show a $\times 2$ fading at NIR wavelengths between 1983 and 1996; assuming a constant rate of fading, this represents only a 0.08 mag fading between the 2MASS (June 2000) and 
KI epochs (June, October 2002), un-important for our purposes. We nevertheless augment the 2MASS errors by $0.1 \mathrm{mag}$ to allow for this level of variation, as well as flickering known to exist in similar systems (see e.g. van den Ancker et al. 2004; Kenyon et al. 2000). Thermal-IR data is from ISOPHOT as presented by Abraham et al. (2004).

V1515 Cyg: Same as above, except that: (1) no I-band data is available, and (2) given the large ISOPHOT beam and beam confusion issues discussed for this object by Abraham et al. (2004), we use long-wavelength fluxes from the aperture photometry in Kenyon, Hartmann \& Kolotilov (1991). We note that the level of NIR fading reported by Abraham et al. (2004) is much less significant even than for v1057 Cyg. The epoch of Maidanak UBVR data is June 2003; the epoch of JHK 2MASS data is November 1998.

Z CMa-SE: Composing an SED for the FU Ori component in this system is further complicated by the fact that most existing photometry is for the total system. For UBV, we use the near-contemporaneous Maidanak photometry (epoch January 2004), given that at these wavelengths the FU Ori component is thought to dominate the total flux (see e.g. Thiebaut et al. 1995). An estimate of the R-band flux is obtained from the Maidanak photometry using the flux ratio of Thiebaut et al. (1995). Infrared J \& H fluxes are estimated by combining the 2MASS total fluxes (epoch December 1998) and the flux ratios from the spatially resolved observations of Millan-Gabet \& Monnier (2002) (epoch January 2001). At longer wavelengths, the infrared fluxes are from the discovery observations of Koresko et al. (1991) (epoch Novemeber 1990). The K-band flux has been dimmed by 0.2 mag in order to account for the fading observed by van den Ancker et al. (2004).

\subsection{Accretion Disk Models}

Accretion disk models have proved successful at reproducing many of the observational properties of FU Orionis objects (Hartmann \& Kenyon 1985, 1996, and references therein). A key prediction of accretion disk models is the shape of the broadband SEDs, and agreement with observations provide one of the strongest lines of support for the disk hypothesis. For a steady Keplerian disk that is optically thick and geometrically thin, its radial temperature profile can be parametrized as (Shakura \& Sunyaev 1973; Lynden-Bell \& Pringle 1974): $T(r)=T_{\max } \cdot\left(r / R_{\star}\right)^{q=-3 / 4} \cdot\left[1-\left(R_{\star} / r\right)^{1 / 2}\right]^{1 / 4}$, for $r>1.36 R_{\text {star }}$, and $T(r)=T_{\text {max }}$ between this radius and the stellar surface. The maximum temperature in the disk $\left(T_{\max }\right)$ is related to the stellar mass and disk accretion rate, and good SED fits are obtained with relatively high values of this parameter $\sim 5000-7000 \mathrm{~K}$, together with stellar radii normal for low-mass 
young stars $2-4 R_{\odot}$. Under these conditions, the disk flux far outshines the central $\operatorname{star}^{2}$ at all wavelengths and the model has relatively few parameters. If the disk inclination can be reasonably well constrained by other means, then the extinction $A_{V}$, stellar radius $R_{\star}$ and maximum disk temperature $T_{\max }$ are all well constrained by the shape and level of the SED. Once a model solution is thus obtained, the corresponding visibilities can be computed and a straightforward comparison to the KI data be made.

Another characteristic of these single power-law models is that, except for inclinations effects (believed to be low for our sources, e.g. Kenyon, Hartmann \& Hewett (1988)), flux and "size" at a given wavelength are simply related. This scaling, and comparison with the FU Ori Palomar Testbed Interferometer (PTI) observations of Malbet et al. (1998), immediately reveals that our targets are over-resolved with respect to expectations from the disk model. Indeed, our observations of V1057 Cyg and V1515 Cyg are approximately consistent with the visibility curve for FU Ori in Malbet et al. (1998), corresponding to a disk solution which also fits its SED. However, V1057 Cyg and V1515 Cyg are $\sim \times 38$ and $\times 8$ fainter than FU Ori, respectively. Therefore, a disk model that fits the SEDs of V1057 Cyg and V1515 Cyg will clearly under-estimate their visibilities.

Following previous workers, our disk is represented by a series of concentric annuli. At each radius, the annulus radiates as a Kurucz (Kurucz 1979) supergiant photosphere of effective temperature equal to the disk temperature at that radius, unless the temperature is lower than $3500 \mathrm{~K}$, in which case we use blackbody emission. We use solar metallicity and a value of the surface gravity appropriate for the temperature at each radius. Model SEDs are reddened using the law of Mathis (1990), using a total-to-selective extinction ratio $R_{V}=3.1$. The KI data were obtained essentially at a single baseline position angle and therefore can not constrain the disk inclination. Moreover, for V1057 Cyg and V1515 Cyg, the rotational velocities measured from line broadening are relatively low, such that unless the stars have unreasonably low mass, the inclinations must be low $\left(i<30^{\circ}\right.$, Kenyon, Hartmann \& Hewett 1988). Therefore we consider mainly face-on disks in our fits, but explore the effects of disk inclination.

The solutions found from fitting the SEDs are similar to those found by previous workers, and are shown in Table 5. The data and disk models are shown in Figure 1. For V1057 Cyg, in this and subsequent visibility figures, we include (open symbol) the data obtained at the PTI by Wilkin \& Akeson (2003). However, due to the very different FOVs of the KI and PTI instruments, and given the possible effect of large scale emission in these objects (see $\S 3.4$ )

\footnotetext{
${ }^{2}$ We note that SED solutions with much lower $T_{\max }$ and larger $R_{\star}$ are possible, but require essentially all the $\mathrm{U} \rightarrow \mathrm{R}$ flux to arise in the stellar photosphere, which is in conflict with the measured line profiles.
} 
we choose not to include this datum in our fitting exercises. In the visibility panels it can be clearly seen that geometrically flat accretion disks (i.e. single power law with $q=-0.75$ ) significantly overestimate the visibility amplitudes at K-band, because the relatively steep temperature profile results in the NIR emission coming from regions of the disk that have too small radii.

As is well known, and as is also apparent from the SED panels of Figure 1, for some FU Orionis objects (such as V1057 Cyg and V1515 Cyg) longward of 10 $\mathrm{m}$ the infrared fluxes far exceed the flat disk predictions. Flared disks having a flatter temperature profile have been proposed as one solution to explain the long-wavelength SED (see e.g. Kenyon \& Hartmann 1991; Lachaume 2004). We have approximated this model by specifying a double-power law for the temperature profile in the disk. Following Kenyon \& Hartmann (1991), we use Figure 4 of their paper, and select the case of most extreme flaring $\left(H_{0}=0.2\right.$, this parameter determines the disk height profile as a function of radius, $H_{d}(r) / R_{\star}=H_{0}$. $\left.\left(r / R_{\star}\right)^{9 / 8}\right)$, to approximate their detailed temperature profile with two slopes: $q=-0.75$ for $r<100 \cdot R_{\star}$ and $q_{2}=-0.45$ outside this radius. Figure 1 shows that for flared disks the long wavelength SED is now well reproduced; however the K-band visibilities are indistinguishable from the non-flared case, simply because the flared disk regions are too cool to thermally emit significant NIR radiation.

For Z CMa-SE, there exists no resolved long wavelength photometry, and therefore we consider only the flat disk model. This source is much more resolved than the other two, at levels very difficult to explain with thermal disk emission only.

We have explored two additional possibilities to attempt to explain the low visibilities measured by KI using purely thermal disk emission: disk inclination and a non-standard temperature profile $(q \neq-0.75)$ for the inner-disk, as follows:

The SEDs can be well fit for non-zero inclinations, provided the flux lost to surface area is recovered by increasing $R_{\star}$; which also has the desired effect of resulting in lower visibilities (a larger NIR source) if the KI baseline position angle also happens to be aligned with a long direction of the elliptical brightness. However, we find that, even allowing for the most favorable disk orientation, the inclinations needed to match the KI data are very large, $i \sim 60-85^{\circ}$, well outside the upper limit inferred from line broadening for typical young star masses range, as mentioned above. We illustrate this difficulty for V1057 Cyg in the top panel of Figure 2, and similar conclusions apply to V1515 Cyg.

Relaxing the assumption that $q=-3 / 4$ and allowing a flatter profile in the inner disk would also result in larger NIR emission regions. Exploring this departure from the canonical temperature profile exponent is a reasonable approach, given that (a) the decaying and 
flickering light curves show that the disk is not exactly steady-state, and (b) the absence of the expected boundary layer emission (Kenyon \& Hartmann 1989) calls into question the radial temperature profile near the inner disk edge. We find however that matching the visibilities would require $q \geq-0.6$, and for such exponents the SED can not be satisfactorily fit, because of the sensitivity of its infrared slope to the temperature profile exponent. Allowing a free q-parameter and the disk to be inclined does not provide a satisfactory solution either, simply because $q \sim-0.65$ is the flattest exponent allowed by the NIR SED slope, and for such exponents large disk inclinations would again be needed to match the visibilities. We illustrate these difficulties for V1057 Cyg in the bottom three panels of Figure 2, and similar conclusions apply to V1515 Cyg.

Finally, we note that allowing the disk inner radius to be a free parameter $\left(r_{\text {inner }}>>R_{\star}\right.$ can formally result in acceptable simultaneous fits to the SEDs and visibilities, but we believe are not physically viable for our targets (contrary to the case of Herbig Ae/Be and T Tauri systems). Allowing an inner disk radius several times larger than $R_{\star}$ (for example $10 \times R_{\star}$ for V1057 Cyg), and keeping the same $T_{\max }$ to preserve the SED shape, results in NIR emission originating at larger radii (comparable to the ring radii of $\S 3.1$ ), and lower visibilities in agreement with the observations. However, to preserve the SED flux levels, the disk needs to be inclined by very large amounts $\left(86^{\circ}\right.$ for V1057 Cyg), in contradiction to the limits derived by Kenyon, Hartmann \& Hewett (1988). Moreover, physical disk models (Clarke, Lin \& Pringle 1990; Hartmann, Kenyon \& Calvet 1993; Bell \& Lin 1994) indicate that for the high accretion rates of FU Ori systems, it is very implausible that a central optically thin disk region could exist; and the emission lines that would be expected if it did are not observed.

\subsection{Additional Model Components}

The difficulties encountered in the previous section in using standard disk models to simultaneously reproduce the observed SEDs and visibilities may indicate that these models

are inadequate for describing the NIR emission. Alternatively, additional source components could exist which are responsible for the low measured visibilities.

\subsubsection{Extended Structure}

A possible explanation is that the measured visibilities are not entirely due to compact NIR thermal emission in a flat or flared disk, but that small but non-negligible fraction of the 
total flux arises in larger scale structure within the 50 mas FOV of the interferometer (25 AU and $50 \mathrm{AU}$ at $d=500,1000 \mathrm{pc}$ respectively). The size of this region and the flux it contributes are degenerate when modelling its contribution to the visibility. However, a minimum flux can be inferred if we assume that it is completely incoherent (i.e. completely resolved by the interferometer), which for the spatial frequencies sampled and the distances to the three objects corresponds to size scales $\gtrsim 4$ AU, 5 AU, and 10 AU, for V1057 Cyg, V1515 Cyg and ZCMa-SE respectively. The needed amount of incoherent flux can be analitically estimated as follows. For an object consisting of a central compact object (the NIR disk, we neglect the stellar flux) plus an extended component, the measured visibility (at a given spatial

frequency) is: $V_{\text {measured }}=\left(F_{C} \cdot V_{C}+F_{E} \cdot V_{E}\right) / F_{T}$; where the subscripts in the flux $(F)$ and visibility $(V)$ of each component denote: $C=$ Compact, $E=$ Extended and $T=$ Total. In the case that the extended component is completely resolved, we have $V_{E}=0.0$, and the minimum incoherent flux fraction is given by: $f_{i}=F_{E} / F_{T}=1.0-V_{\text {measured }} / V_{C}$. Table 5 summarizes the values for $f_{i}$ obtained for the cases that the compact object is unresolved $\left(V_{C}=1.0\right)$ and, more meaningfully, the case that the compact object is the accretion disk considered in the previous section, and is resolved at the data spatial frequencies by the amounts predicted by the best-SED-fit models (Table 5 and Figure 1). The effect of this additional component on the visibility curves is shown in Figure 3, where the ranges of $f_{i}$ shown correspond to the values needed to reproduce the weighted mean visibility measured, and visibility data upper limits given the $+1 \sigma$ errors.

\subsubsection{Physical Origin of the Extended Emission}

Given the low thermal temperatures expected for any circumstellar material located at the scales considered above, physically this additional emission would correspond to scattering of thermal disk K-band photons by circumstellar material (at least for V1057 Cyg and V1515 Cyg, the fraction of incoherent flux derived above for ZCMa-SE is prohibitely high for a scattering origin). Our observations do not directly constrain the precise nature of the putative incoherent flux source, reasonable hypothesis are that scattering occurs in the upper atmosphere of the outer disk, or in envelope material.

Kenyon \& Hartmann (1991) first showed that thermal emission in infalling envelopes surrounding the V1057 Cyg and V1515 Cyg disks provide good fits to the long wavelength excess fluxes in these systems. The putative envelopes have evacuated cones along the poles, cleared by bipolar outflows, which result in the relative un-obscured views towards the central objects, as observed. Moreover, these authors favor the envelope hypothesis over flaring disks, due to the large degree of flaring that would be required to reproduce the long- 
wavelength SEDs, and the wavelength dependent photometric decay curves. For T Tauri objects with flat SEDs, Natta (1993) have also proposed the existence of envelopes (tenous in that case) which heat the outer disk regions by scattering stellar photons onto it.

The envelopes considered by Kenyon \& Hartmann (1991) for V1057 Cyg and V1515 Cyg are characterized by $\sim 7 \mathrm{AU}$ inner radii and temperatures at these radii of $\sim$ few $100 \mathrm{~K}$; and it is therefore easy to see that adding such an envelope to the disk has no effect on the K-band visibilities if only thermal emission is considered; as pointed out by these authors the emission below $10 \mu \mathrm{m}$, and certainly at $2 \mu \mathrm{m}$, is completely dominated by the disk. Moreover, the $\sim 7$ AU inner radii imply that this component would indeed be completely resolved by the KI. Therefore, the basic considerations presented in $\S 3.4 .1$ provide the amounts of flux that the envelope would need to scatter at K-band to reproduce the KI observations.

These are relatively large amounts of scattered K-band flux, originating in a relatively small FOV. However, building upon the initial models of Kenyon \& Hartmann (1991), more refined models of V1057 Cyg and V1515 Cyg are being explored and compared with new data from the Infrared Spectrograph on board the Spitzer Space Telescope, which involve infall to the disk at about $10 \mathrm{AU}$ (Green et al. 2005). Independent of model details, the long-wavelength excesses above what is expected for the disk are approximately $20 \%$ of the apparent total disk luminosity for V1057 Cyg and 12\% of the total apparent luminosity of V1515 Cyg. If the effective scattering albedo of the envelope in the $\mathrm{K}$ band is of order of 0.5, then the envelopes invoked to explain the infrared excesses longward of $\sim 10 \mu \mathrm{m}$ could be responsible for a scattered flux of $6-10 \%$ within the FOV of the interferometer. This is in reasonable agreement with the observational requirements, given the uncertainties and likely complex geometry, implying that the scattering envelopes of V1057 Cyg and V1515 Cyg can in fact account for the K-band visibilities.

\subsubsection{Stellar Companion}

Dynamical interactions with a stellar companion have been invoked to explain the origin of the accretion rate outbursts in FU Ori objects (Bonnell \& Bastien 1992), and in fact several objects of the class are known to have "wide" (tenths of arcsec separations) companions. Motivated by the recent discovery of such a wide companion (separation $\sim 0.5^{\prime \prime}$ ) to FU Ori itself (Wang et al. 2004), Reipurth \& Aspin (2004) propose that the FU Ori phenomenon corresponds to the formation of binary systems by break up of small nonhierarchical systems. For FU Ori itself, this scenario predicts a third close companion, at separation $\sim 10 \mathrm{AU}$, perhaps corresponding to the interferometric signature detected by Malbet et al. (2005). 
A binary system produces a sinusoidal visibility amplitude curve as a function of projected baseline, with period given by the (inverse of) angular separation and an amplitude given by the flux ratio between the two components. Given our limited data set (essentially a single spatial frequency was measured for each object), and the sinusoidal response, many values of the binary parameters (flux ratio and companion location) result in visibility functions that reproduce our measurements equally well, and therefore a fitting excercise is not very informative. However, for reference, interesting bounds on the family of possible solutions are as follows. The measured visibility values imply a maximum flux ratio between the binary components of about: 20:1, 20:1 and 2.5:1 for V1057 Cyg, V1515 Cyg and Z Cma-SE respectively. For this maximum value of the flux ratio, the minimum angular separations are about: 1.0, 1.0 and 1.7 mas for V1057 Cyg, V1515 Cyg and Z Cma-SE respectively. For equal flux components, the minimum separations become: 0.4, 0.4 and 1.3 mas for V1057 Cyg, V1515 Cyg and Z Cma-SE respectively. We note that for these estimates we have considered a binary model in which the "primary" component is again the partially resolved best-SED-fit disk of $\S 3.3$.

\section{Discussion and Conclusions}

We have presented new observations which spatially resolve the NIR emission of three FU Orionis objects on mas scales, a first for two of the objects (V1515 Cyg and Z CMa-SE). The spatial scales for NIR emission are surprisingly large, compared to predictions of simple accretion disk models. This indicates that the accretion models require modification, or that additional model components are required.

As is well known, the infrared SED slopes sensitively probe the radial temperature profile in the disk, and we have shown that while exponents $q=-0.6$ or flatter would be required to match the measured visibilities, these solutions are not able to maintain good agreement with the SED data. We note however that in this approach, tests of the validity of small variations to the disk temperature profile are limited by the accuracy of the SED photometry adopted, and therefore follow-up studies should ideally be performed using contemporaneous photometry of uniform quality.

It is interesting to contrast our results with the case of the prototype for the class, FU Orionis, which has only modest long wavelength fluxes in excess of thermal emission in a flat accretion disk, and for which the NIR interferometer data does agree well with this model according to Malbet et al. (1998, 2005). We note that this conclusion rests on the ability to incline the FU Ori disk by a relatively large amount $\left(i \sim 50-60^{\circ}\right)$, which in this

case does not imply unreasonably low stellar masses given the measured rotational velocities 
(Kenyon, Hartmann \& Hewett 1988).

Given the existing indications for dense envelopes around V1057 Cyg and V1515 Cyg, we hypothesize as a natural explanation for the low measured visibilities that scattering by material located on 10s of AU scales adds incoherent flux in the interferometer FOV, diluting the visibilities from the relatively compact inner disk. We note that this mechanism may also explain the somewhat larger size (Gaussian FWHM = 1.36 \pm 0.07 mas) found for V1057 Cyg by Wilkin \& Akeson (2003), since the Palomar Testbed Interferometer has a $1^{\prime \prime}$ FOV, $\times 20^{2}$ larger than that of the KI. A full calculation of FOV effects in specific scattering models is beyond the scope of this paper, we simply note that considering the PTI measurement alone, the minimum incoherent flux fraction becomes $f_{i}=21 \pm 5 \%$.

An important consequence of this interpretation is that it compromises the notion to use FU Orionis objects as straightforward test cases of accretion disk theories, at least for most of the better known candidate targets. Indeed, if the disk is not isolated, multi-baseline and multi-wavelength interferometer observations must be used to better discriminate between competing models. In particular, the hypothesis that scattering is at the origin of the low visibilities measured is testable using measurements in different NIR bands: at shorter wavelengths were scattering is more efficient (e.g. J or H bands) the characteristic sizes should be larger; while thermal disks predict the opposite trend of larger sizes at longer wavelengths (and with a detailed dependence that probes the flatness of the temperature profile). These multi-wavelength observations are already possible at existing interferometers (e.g. KI and the AMBER instrument on the VLTI, Petrov et al. (2000)) or upcoming instruments (e.g. the MIRC combiner at CHARA, Monnier et al. (2004)). For objects with evidence for dense envelopes, self-consistent radiative transfer codes that include scattering should also be used in order to include this effect in both the SEDs and visibilities for realistic geometries and dust properties.

The exciting possibility that all three objects are instead resolved due to the presence of stellar companions, sometimes invoked to explain the outburst themselves, is also testable with follow-up interferometer observations with improved spatial frequency coverage. Given the KI visibility calibration accuracy (which translates into a 100:1 maximum detectable binary contrast), the telltale and un-ambiguous sinusoidal signatures would be easily detected even for the most pessimistic values of the binary contrast derived above.

The authors wish to thank the all members of the Keck Interferometer development team (JPL, MSC, WMKO) whose dedicated efforts made this "shared-risk" commissioning science possible. We also thank Dr. Francis Wilkin for providing the PTI data for V1057 Cyg. This material is based upon work supported by NASA under JPL Contracts 1236050 \& 1248252 
issued through the Office of Space Science. Data presented herein were obtained at the W.M. Keck Observatory from telescope time allocated to the National Aeronautics and Space Administration through the agency's scientific partnership with the California Institute of Technology and the University of California. The Observatory was made possible by the generous financial support of the W.M. Keck Foundation. This research has made use of the SIMBAD database, operated at CDS, Strasbourg, France. This publication makes use of data products from the Two Micron All Sky Survey (2MASS), which is a joint project of the University of Massachusetts and the Infrared Processing and Analysis Center/California Institute of Technology, funded by the National Aeronautics and Space Administration and the National Science Foundation. This work has made use of services produced by the Michelson Science Center at the California Institute of Technology. The authors wish to recognize and acknowledge the very significant cultural role and reverence that the summit of Mauna Kea has always had within the indigenous Hawaiian community. We are most fortunate to have the opportunity to conduct observations from this mountain.

\section{REFERENCES}

Abraham, P.; Kospal, A.; Csizmadia, Sz.; Kun, M.; Moor, A.; Prusti, T. 2004, A\&A, 428, 98

Akeson, R. L., Ciardi, D. R., van Belle, G. T., Creech-Eakman, M. J., and Lada, E. A. 2000, ApJ, 543, 313, Paper 1

Akeson, R. L., Ciardi, D. R., van Belle, G. T., \& Creech-Eakman, M. J. 2002, ApJ, 566, 1124

Akeson, R. L. et al. 2005, ApJ, 622, 440

Akeson, R. L. et al., 2005, ApJ, 635, 1173

Bell, K. R. and Lin, D. N. C. 1994 ApJ, 427, 987

Bonnell, I. and Bastien, P. 1992, ApJ, 401, L31

Clarke, C. J., Lin, D. N. C. and Pringle, J. E. 1990, MNRAS, 242, 439

Clarke, C., Lodato, G., Melnikov, S.Y., and Ibrahimov, M.A. 2005, MNRAS, in press

Colavita, M. M.; Wizinowich, P. L.; Akeson, R. L. 2004, in Proceedings of SPIE Volume 5491, p. 454 
Colavita, M. M. et al. 2003, ApJ, 529, 83L

Colavita, M. M. 1999, PASP, 111, 111

Dullemond, C. P.; Dominik, C.; Natta, A. 2001, ApJ, 560, 957

Eisner, J.A., Lane B.F., Hillenbrand, L.A., Akeson, R.L. \& Sargent, A.I., 2004, ApJ, 613, 1049

Eisner, J.A., Hillenbrand, L.A., White, R.J. Akeson, R.L. \& Sargent, A.I., 2005, ApJ, in press

Garcia, P. J. V.; Thiebaut, E.; Bacon, R. 1999, A\&A, 346, 892

Goodman, J. W. 1985, "Statistical Optics", John Wiley \& Sons

Green, J. et al. 2005, in preparation

Hartmann, L.; Kenyon, S. J. 1985, ApJ, 299, 462

Hartmann, L.; Kenyon, S. J.; Hewett, R.; Edwards, S.; Strom, K. M.; Strom, S. E.; Stauffer, J. R. 1989, ApJ, 338, 1001

Hartmann, L., Kenyon, S. J. and Calvet, N. 1993, ApJ, 407, 219

Hartmann, L. and Kenyon, S. J. 1996, ARA\&A, 34, 207

Hartmann, L.; Hinkle, K.; Calvet, N. 2004, ApJ, 609, 906

Herbig, G. H.; Petrov, P. P.; Duemmler, R. 2003, ApJ, 595, 384

Herbst, W.; Racine, R.; Warner, J. W. 1978, ApJ, 223, 471

Herbst, W. and Shevchenko, V.S. 1999, AJ, 118, 1043

Kenyon, S.; Hartmann, L. 1989, ApJ, 324, 1134

Kenyon, S. J.; Hartmann, L.; Hewett, R. 1988, ApJ, 325, 231

Kenyon, S. J.; Hartmann, L. W.; Kolotilov, E. A. 1991a, PASP, 103, 1069

Kenyon, Scott J.; Hartmann, Lee W. 1991b, ApJ, 383, 664

Kenyon, Scott J.; Kolotilov, E. A.; Ibragimov, M. A.; Mattei, Janet A. 2000, ApJ, 531, 1028 
Kopatskaya, E. N.; Grinin, V. P.; Shakhovskoi, D. N.; Shulov, O. S. 2002, Astrophysics, 45, 2,143

Koresko, C. D. et al. 1991, AJ, 102, 2073

Kurucz, R. L. 1979, ApJS, 40, 1

Larson, R. B. 1980, MNRAS, 190, 321

Lachaume, R. 2004, A\&A, 422, 171

Lynden-Bell, D.; Pringle, J. E. 1974, MNRAS, 168, 603L

Malbet, F. et al. 1998, ApJ, 507, 149L

Malbet F. et al. 2005, A\&A, in press

Mathis, J. S. 1990, ARA\&A, 28, 37

Millan-Gabet, R., Schloerb, F. P., Traub, W. A., Malbet, F., Berger, J. P., \& Bregman, J. D. 1999, ApJ, 513, L131

Millan-Gabet, R., Schloerb, F. P., and Traub, W. A, 2001, ApJ, 546, 358

Millan-Gabet, R.; Monnier, J. D. 2002, ApJ, 580, 167L

Millan-Gabet, R., Malbet, F., Akeson, R., Leinert, Ch., Monnier, J. D., Waters, R. 2006, in Protostars and Planets V, Eds. Reipurth B., Jewitt, K. \& Keil, K., Univ. of Arizona Press (Tucson), in press

Monnier, J. D.; Millan-Gabet, R. 2002, ApJ, 579, 694

Monnier, John D.; Berger, Jean-Philippe; Millan-Gabet, Rafael; Ten Brummelaar, Theo A. 2004, in Proceedings of SPIE Volume 5491, p. 1370

Monnier, J. D. et al. 2005, ApJ, 624, 832

Muzerolle, J.; Calvet, N.; Hartmann, L.; D’Alessio, P. 2003, ApJ, 597, 149

Natta, A.; Prusti, T.; Neri, R.; Wooden, D.; Grinin, V. P.; Mannings, V. 2001, A\&A, 371, 186

Natta, A., 1993, ApJ, 412, 761

Petrov, R. et al. 2000, in Proc. SPIE Vol. 4006, p. 68-79 
Racine, R. 1968, AJ, 73, 588

Reipurth, B. and Aspin, C. 2004, ApJ, 608, L65

Shakura, N. I.; Sunyaev, R. A. 1073, A\&A, 24, 337

Shao, M. \& Staelin, D. H. 1977, Optical Society of America Journal, vol. 67, p. 81

Straizys, V.; Meistas, E.; Vansevicius, V.; Goldberg, E. P. 1989, A\&A, 222, 82

Swain, M. 2003, ApJ, 596L, 163

Thiebaut, E.; Bouvier, J.; Blazit, A.; Bonneau, D.; Foy, F.-C.; Foy, R. 1995, A\&A, 303, 795

Tuthill, P. G.; Monnier, John D.; Danchi, William C. 1999, Nature, 398, 487

van den Ancker, M. E.; Blondel, P. F. C.; Tjin A Djie, H. R. E.; Grankin, K. N.; Ezhkova, O. V.; Shevchenko, V. S.; Guenther, E.; Acke, B. 2004, MNRAS, 349, 1516

Vinković, D. et al. 2003, MNRAS, 346, 1151

Wang, H.; Liu, C.; Qiu, J.; Deng, N.; Goode, P. R.; Denker, C. 2004, ApJ, 601, L83

Wilkin, Francis P.; Akeson, Rachel L. 2003, Astrophysics and Space Science, v. 286, Issue 1, p. 145

Wizinowich, P. L. et al., 2003, in Proceedings of the SPIE, Volume 4839, p. 9 
Table 1. Basic Properties of Targets.

\begin{tabular}{cccccccccc}
\hline \hline Name & $\begin{array}{c}\text { RA } \\
(\mathrm{J} 2000)\end{array}$ & $\begin{array}{c}\text { Dec } \\
(\mathrm{J} 2000)\end{array}$ & $\begin{array}{c}\mathrm{V} \\
(\mathrm{mag})^{\mathrm{a}}\end{array}$ & $\begin{array}{c}\mathrm{J} \\
(\mathrm{mag})^{\mathrm{a}}\end{array}$ & $\begin{array}{c}\mathrm{H} \\
(\mathrm{mag})^{\mathrm{a}}\end{array}$ & $\begin{array}{c}\mathrm{K} \\
(\mathrm{mag})^{\mathrm{a}}\end{array}$ & $\begin{array}{c}\mathrm{d} \\
(\mathrm{pc})\end{array}$ & $\begin{array}{c}\text { Adopted } \\
T_{\star}(\mathrm{K})^{\mathrm{b}}\end{array}$ & $\begin{array}{c}\text { SED } \\
\text { photometry references }^{\mathrm{c}}\end{array}$ \\
\hline V1057 Cyg & 205853.7 & +441528.4 & 11.7 & 8.0 & 7.0 & 6.2 & $550 \pm 100(1)$ & 6200 & $(4,5,6,7)$ \\
V1515 Cyg & 202347.6 & +421224 & 12.4 & 8.9 & 8.0 & 7.4 & $1000 \pm 200(2)$ & 5845 & $(5,7,8)$ \\
Z CMa-SE & 070343.2 & -113306.2 & 9.8 & 6.5 & 5.2 & 3.8 & $1150(3)$ & 6360 & $(5,7,9,10)$ \\
\hline
\end{tabular}

${ }^{a}$ As discussed in the text, these objects are by defition highly variable. The $\mathrm{V}$ and infrared magnitudes in this table are from SIMBAD and 2MASS respectively, and are intended to be merely representative. See the text and references in this table for a description of how the SED used in the data analysis have been constructed. For Z CMa the magnitudes given in this table are for the total system.

${ }^{\mathrm{b}}$ The true photospheric spectral types are not known as there are no pre-outburst spectra of these objects, these values are typical of what is quoted in the literature. The exact photospheric effective temperatures are however inconsequential to the analysis given the negligible stellar flux contribution in the models considered.

${ }^{\mathrm{c}}$ As indicated in the text, we use near-contemporaneous UBVR photometry obtained at Maidanak Observatory (ROTOR program). In this column we provide the relevant references for additional literature photometry used to construct our SEDs.

References. - (1) Straizys et al. (1989); (2) Racine (1968); (3) Herbst, Racine \& Warner (1978); (4) Kopatskaya et al. (2002); (5) 2MASS All-Sky Point Source Catalog; (6) Abraham et al. (2004); (7) van den Ancker et al. (2004); (8) Kenyon, Hartmann \& Kolotilov (1991); (9) Millan-Gabet \& Monnier (2002); (10) Koresko et al. (1991); 
Table 2. Calibrator Stars Information.

\begin{tabular}{cccccc}
\hline \hline Name & V & J & K & Spectral Type & UD diameter (mas) \\
\hline HD 199178 & 7.2 & 5.7 & 5.2 & G2V & $0.44 \pm 0.07$ \\
HD 192985 & 5.8 & 5.0 & 4.8 & F5V & $0.43 \pm 0.06$ \\
HIP 102667 & 8.8 & 6.4 & 5.5 & K2V & $0.49 \pm 0.16$ \\
HD 227049 & 9.1 & 7.0 & 6.3 & K2III & $0.30 \pm 0.10$ \\
HD 332518 & 9.2 & 6.9 & 6.2 & K5V & $0.38 \pm 0.08$ \\
HD 52919 & 8.3 & 6.4 & 5.7 & K5V & $0.42 \pm 0.09$ \\
HD 48286 & 7.1 & 5.9 & 5.5 & F7V & $0.35 \pm 0.08$ \\
HD 60491 & 8.2 & 6.5 & 6.0 & K2V & $0.33 \pm 0.05$ \\
\hline
\end{tabular}


Table 3. Calibrated Visibility Amplitude Data.

\begin{tabular}{ccccccc}
\hline \hline Name & U.T. Date & Julian Date & $\mathrm{u}(\mathrm{m})$ & $\mathrm{v}(\mathrm{m})$ & $V^{2}$ & Calibrators \\
\hline V1057 Cyg & Jun 27 2002 & 2452453 & 44.519 & 67.770 & $0.727 \pm 0.161$ & HD199178, HD192985, HIP102667 \\
V1057 Cyg & Oct 24 2002 & 2452572 & 43.427 & 70.711 & $0.791 \pm 0.093$ & HD199178, HD199998 \\
V1515 Cyg & May 21 2003 & 2452781 & 54.973 & 55.878 & $0.840 \pm 0.030$ & HD227049, HD332518 \\
V1515 Cyg & May 21 2003 & 2452781 & 53.245 & 59.776 & $0.725 \pm 0.087$ & HD227049, HD332518 \\
V1515 Cyg & May 21 2003 & 2452781 & 51.096 & 63.262 & $0.707 \pm 0.065$ & HD227049, HD332518 \\
Z CMa-SE & Apr 03 2004 & 2453099 & 24.195 & 51.929 & $0.158 \pm 0.016$ & HD48286, HD52919, HD60491 \\
Z CMa-SE & Apr 03 2004 & 2453099 & 23.753 & 51.887 & $0.177 \pm 0.009$ & HD48286, HD52919, HD60491 \\
\hline
\end{tabular}

Note. - This table shows only the statistical $V^{2}$ error, as discussed in $\S 2$ an additional systematic error of 0.05 is added (in quadrature) to all data for fitting purposes and the total error also is shown in all figures. 
Table 4. Characteristic Near-Infrared Sizes.

\begin{tabular}{cccccc}
\hline \hline & \multicolumn{2}{c}{ Gaussian FWHM } & & \multicolumn{2}{c}{ Ring Inner Diameter } \\
\cline { 2 - 3 } \cline { 5 - 6 } Name & (mas) & $(\mathrm{AU})$ & & $(\mathrm{mas})$ & $(\mathrm{AU})$ \\
\hline V1057 Cyg & $1.04 \pm 0.23$ & $0.57 \pm 0.16$ & & $1.11 \pm 0.24$ & $0.55 \pm 0.15$ \\
V1515 Cyg & $1.05 \pm 0.12$ & $1.05 \pm 0.24$ & & $1.13 \pm 0.12$ & $1.13 \pm 0.20$ \\
Z CMa-SE & $3.94 \pm 0.24$ & $4.53 \pm 0.48$ & & $3.81 \pm 0.18$ & $4.38 \pm 0.21$ \\
\hline
\end{tabular}


Table 5. Model parameters.

\begin{tabular}{|c|c|c|c|c|c|c|c|c|}
\hline \multirow[b]{2}{*}{ Name } & \multicolumn{4}{|c|}{ Flat Disk } & \multicolumn{2}{|c|}{ Flared Disk } & \multicolumn{2}{|c|}{ Minimum Incoherent Fluxes } \\
\hline & $q$ & $T_{\max }(\mathrm{K})$ & $R_{\star} / R_{\odot}$ & $A_{V}$ & $q_{2}$ & $r_{0} / R_{\star}$ & $f_{i}(\%)\left(V_{C}=1.0\right)$ & $f_{i}(\%)\left(V_{C}=V_{\text {Disk }}\right)$ \\
\hline v1057 Cyg & -0.75 & 7280. \pm 191 & $2.4 \pm 0.1$ & 4.2 & -0.45 & 100. & $12 \pm 5$ & $9 \pm 5$ \\
\hline v1515 Cyg & -0.75 & 8014. \pm 280 & $2.1 \pm 0.2$ & 3.0 & -0.45 & 100. & $10 \pm 2$ & $9 \pm 2$ \\
\hline Z CMa-SE & -0.75 & 5785. \pm 351 & $7.7 \pm 0.6$ & 1.9 & $\ldots$ & $\cdots$ & $59 \pm 3$ & $58 \pm 3$ \\
\hline
\end{tabular}




\section{Disk models}
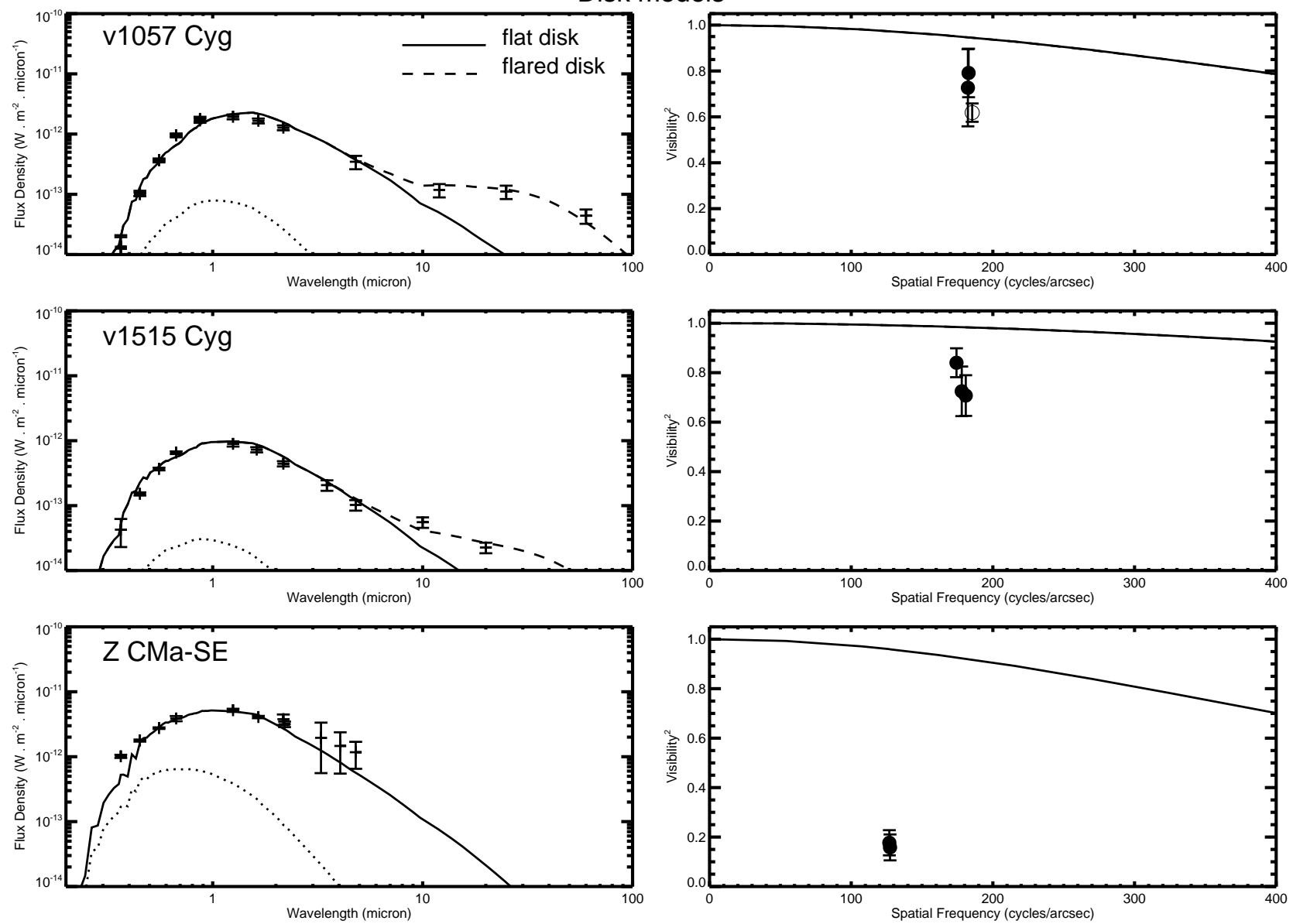

Fig. 1.- Data and disk models results, SED and visibility amplitudes in left and right panels respectively. For V1057 Cyg we include the visibility data obtained at the PTI by Wilkin \& Akeson (2003) (open symbol). The models correspond to best-SED-fits and are calculated for the parameters of Table 5. The solid line shows the model for a flat disk, the dashed line shows the model for a flared disk. For the flat disk model, the fitting procedure uses only SED data with $\lambda<10 \mu m$; while fitting the flared disk model uses the whole SED. In the visibility panels the two lines overlap because the flared disk regions do not contribute significant K-band flux. The dotted line in the SED panels is the stellar photosphere. For Z CMA-SE, we consider only the flat disk model, given the lack of resolved long wavelength photometry. 

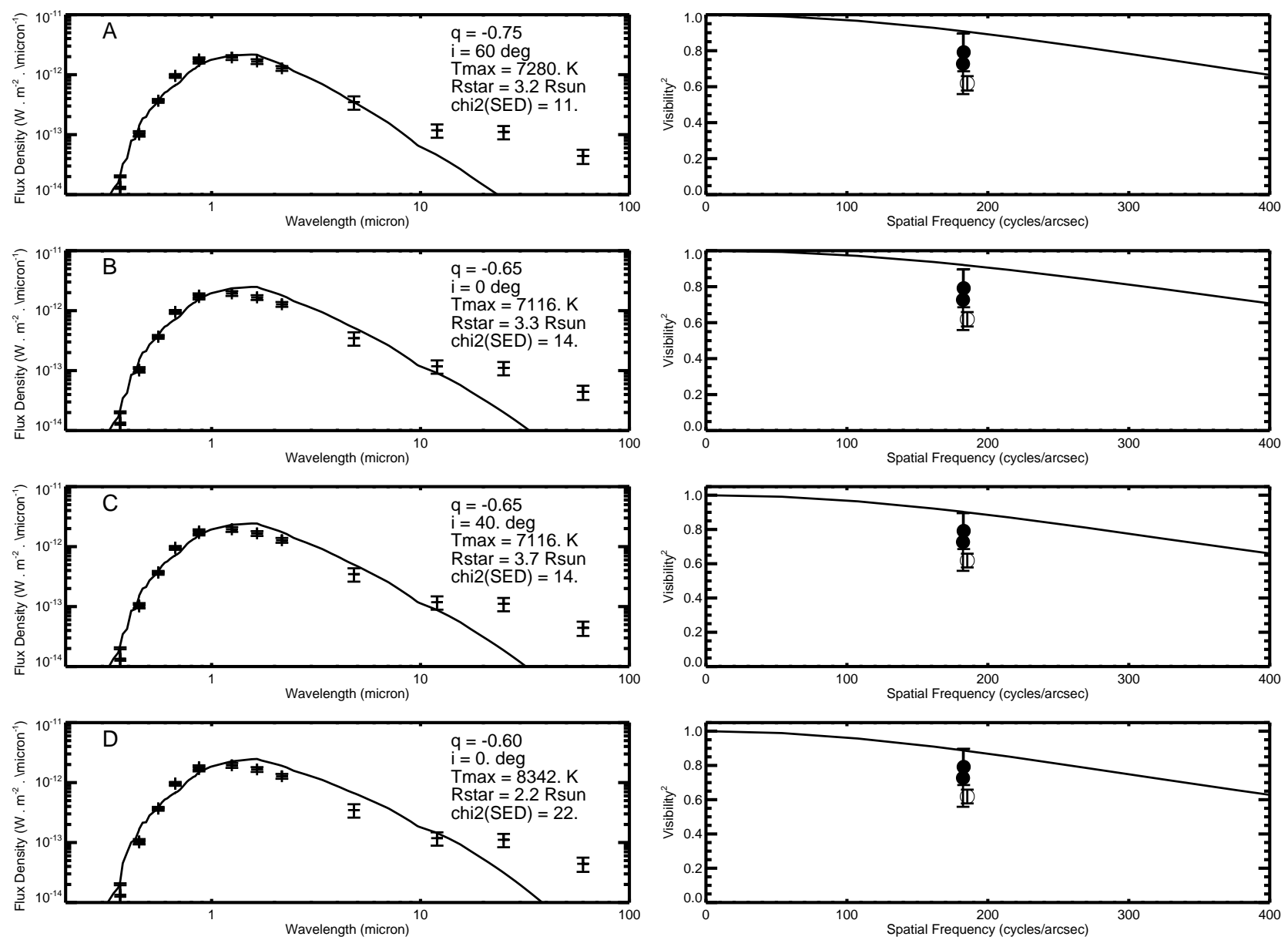

Fig. 2.- V1057 Cyg: Effect of disk inclination (A) and different values of the temperature radial exponent $(\mathrm{B} \rightarrow \mathrm{D})$ for the inner-disk. These models may be compared with the V1057 Cyg best fit (zero inclination) of Figure 1, for which we have the reference reduced $\chi_{S E D}^{2}=11$. Panels A: show that although a $60^{\circ}$ inclination reproduces the SED equally well, it is the minimum inclination required to just become consistent with the upper limit of the $V^{2}$ data. Panels B: show that $q=-0.65$ produces a marginally acceptable SED fit, but is not sufficient to touch the $V^{2}$ upper bound. Panels C: are also for $q=-0.65$, and show that additionally including inclination is able to reproduce the $V^{2}$ upper bound, but a relatively large value of $40^{\circ}$ is required. Panels D: illustrate that $q=-0.6$ reproduce the $V^{2}$ upper bound, even for zero inclination, but results in a significantly poorer SED fit. In all cases, SED data longward of $10 \mu \mathrm{m}$, although plotted, is not included in the calculation of the $\chi_{S E D}^{2}$, since here we only attempt to model the inner disk regions where the K-band flux arises. For V1057 Cyg we include the visibility data obtained at the PTI by Wilkin \& Akeson (2003) (open symbol). 

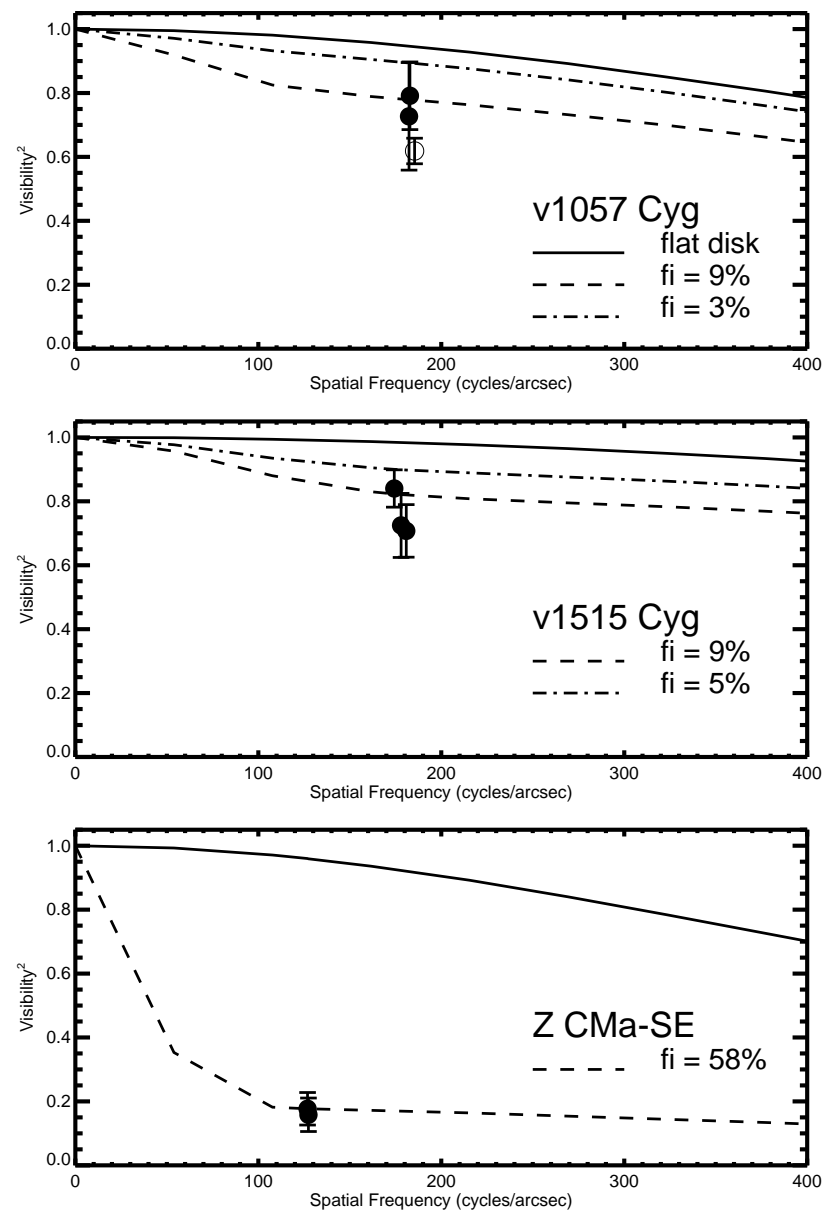

Fig. 3.- Adding incoherent fluxes to the best-SED-fit disk models of Figure 1 (solid line). The dashed lines show how the visibilities are reduced to the desired levels by adding the indicated amounts of incoherent (completely resolved) flux to the disk models. The size of the region where this incoherent flux arises is not constrained by the observations, but for display purposes it has been included in the model as a Gaussian component of FWHM equal to minimum value which is completely resolved at the data spatial frequencies: $4 \mathrm{AU}$, $4 \mathrm{AU}$ and $10 \mathrm{AU}$ for V1057 Cyg, V1515 Cyg and ZCMa-SE respectively. The dotted line in the SED panels is the stellar photosphere. For V1057 Cyg we include the visibility data obtained at the PTI by Wilkin \& Akeson (2003) (open symbol). 